\title{
Multilayer SIW filters with mixed coupling modified trisection (MCMT)
}

\section{Ziqiang $\mathrm{Xu}^{\mathrm{a})}$, Yu Shi, Wanting Zhou, and Peng Gao}

Research Institute of Electronic Science and Technology, University of Electronic Science and Technology of China, Chengdu 611731, China

a)nanterxu@uestc.edu.cn

\begin{abstract}
Mixed coupling modified trisection (MCMT) and its applications to substrate integrated waveguide (SIW) filters are presented. By using source or load to replace a resonator and introducing mixed coupling into conventional trisection, the proposed MCMT not only reduce one resonator but also adds a transmission zero (TZ) to improve the stopand. Moreover, the resonators in MCMT can be embedded into multilayer LTCC substrate to achieve a more compact area. Two SIW filters with two TZs are designed and fabricated to validate the proposed method. Measured results which exhibit good selectivity, agree well with simulated ones.
\end{abstract}

Keywords: LTCC, SIW, filter, MCMT, high selectivity

Classification: Microwave and millimeter wave devices, circuits, and systems

\section{References}

[1] A. Ismail, M. S. Razalli, M. A. Mahdi, R. S. A. R. Abdullah, N. K. Noordin, and M. F. A. Rasid, "X-band trisection substrate-integrated waveguide quasi-elliptic filter,” Prog. Electromagn. Res., vol. 85, pp. 133-145, May 2008.

[2] X. P. Chen and K. Wu, "Self-packaged millimeter-wave substrate integrated waveguide filter with asymmetric frequency response," IEEE Trans. Compon. Packag. Technol., vol. 2, no. 5, pp. 775-782, May 2012.

[3] J. X. Chen, W. Hong, X. P. Chen, P. P. Yan, Q. H. Lai, and K. Wu, “An LTCC X-band receiver front-end using embedded multilayer substrate integrated waveguide filter," Microw. Opt. Techn. Lett., vol. 50, no. 2, pp. 285-287, Feb. 2008.

[4] Q. F. Wei, Z. F. Li, L. Li, W. J. Zhang, and J. F. Mao, “Three-pole crosscoupled substrate-integrated waveguide bandpass filters based on PCB process and multilayer LTCC technology," Microw. Opt. Techn. Lett., vol. 51, no. 1, pp. 71-73, Jan. 2009.

[5] W. Shen, L. S. Wu, X. W. Sun, W. Y. Yin, and J. F. Mao, "Novel substrate integrated waveguide filters with cross coupling (MCC)," IEEE Microw. Wireless Compon. Lett., vol. 19, no. 11, pp. 701-703, Nov. 2009. 


\section{Introduction}

Substrate integrated waveguide (SIW) filters which are synthesized in a planar substrate with arrays of metallic via, could provide a low-profile and easy-integration solution while maintain high performance as conventional metal waveguide filters. To meet the increasing requirements of modern communication systems in terms of compact size and high selectivity, SIW filters with multiple transmission zeros (TZs) using the PCB or LTCC processes have been extensively studied in recent years $[1,2,3,4]$. Crosscoupled SIW filters can obtain additional signal paths to generate TZs at certain frequency. An X-band narrowband trisection elliptic SIW filter with one TZ in [1] and K-band quadruplet SIW filters with two TZs in [2] are proposed to improve frequency selectivity, whereas they are still large due to their numbers of resonators as well as single-layer and planar structures. The application of multilayer LTCC technology makes the realization of cross-coupled SIW filters with compact size, light weight and high performance possible, due to its three dimensional integration characteristic, low-tolerance in manufacturing process, and low loss of high-frequency ceramic materials. In $[3,4]$, X-band LTCC trisection SIW filters with one TZ are introduced. Although the structures in [3, 4] are comparatively compact, their selectivity and upper stopband are needed to improved.

In this paper, novel MCMT structure and its applications to SIW bandpass filters based on LTCC technology are presented to meet the need of stringent frequency selectivity and miniature size. By introducing mixed electric and magnetic coupling into modified trisection, the proposed MCMT could reduce one resonator and generates an additional TZ to improve the frequency selectivity simultaneously. Besides, the resonators in MCMT can be embedded into multilayer substrate to obtain a more compact area. Two SIW filters with MCMT structure are designed and fabricated to validate the proposed method. Measured frequency responses are provided to show good performance and in agreement with the simulated results.

\section{Filter topology and analysis}

The conventional trisection and the proposed MCMT filter are shown in Fig. 1. Compared with the conventional trisection filter in Fig. 1(a), the MCMT filter in Fig. 1(b) could reduce one resonator by using source to replace a resonator while still reserving a $\mathrm{TZ}$ which can flexibly controlled either below or above the passband. Meanwhile, with introducing mixed

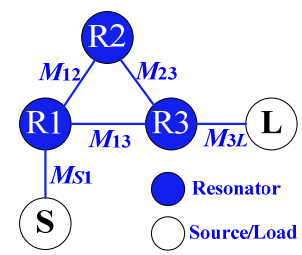

(a)

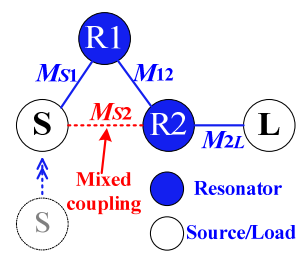

(b)

Fig. 1. Coupling schemes of (a) a conventional trisection and (b) the proposed mixed coupling modified trisection (MCMT). 
coupling (MC) between the source and the resonator 2, another transmission path can be obtained. Under the circumstances, the coexistence of electric and magnetic coupling can be adopted to build dual-coupling paths between source and resonator 2 to introduce an additional $\mathrm{TZ}$, which are usually controllable by adjusting the $\mathrm{MC}$ coefficients. In other words, MC implemented into the proposed topology can also bring another signal path to generate up to two TZs.

The coupling matrix $\mathrm{M}$ of the proposed topology in Fig. 1 (b) can be written as

$$
[M]=\left[\begin{array}{cccc}
0 & M_{S 1} & M_{S 2}(\omega) & 0 \\
M_{S 1} & M_{11} & M_{12} & 0 \\
M_{S 2}(\omega) & M_{12} & M_{22} & M_{2 L} \\
0 & 0 & M_{2 L} & 0
\end{array}\right]
$$

where $M_{S 1}, M_{S 2}$ and $M_{2 L}$ express the coupling between source/load and each resonator, $M_{12}$ represents the coupling between resonators 1 and $2, M_{11}$ and $M_{22}$ of diagonal elements in the coupling matrix symbol the differences in the resonant frequencies of resonators. The position of the TZ due to crosscoupling path can be decided in a low-pass prototype as follows

$$
\Omega=M_{S 1} M_{12} / M_{S 2}(\omega)-M_{11}
$$

where $\Omega=\left(\omega / \omega_{0}-\omega_{0} / \omega\right) / F B W$ is normalized angular frequency while $F B W$ is the fractional bandwidth. $M_{S 2}(\omega)$ represents the $\mathrm{MC}$ between source and resonator 2 which is composed of frequency-variant electric and magnetic coupling, and it can be obtained by using the conventional solution method of a MC coefficient, as described in [5]

$$
M_{S 2}(\omega)=\left[\omega L_{m}-1 /\left(\omega C_{m}\right)\right] / \omega_{0} L
$$

where each resonator in the equivalent circuit of the two mixed coupled resonators is characterized by an inductance $L$ together with a parallel capacitance $C$ and its resonant frequency equals to $\omega_{0}=(L C)^{-1 / 2} \cdot L_{m}$ and $C_{m}$ express the coupling inductance and capacitance and they will produce mixed magnetic and electric coupling, respectively. Besides, the coupling coefficient at center frequency $\omega_{0}$ is written as

$$
k\left(\omega_{0}\right)=L_{m}\left(1-\omega_{m}^{2} / \omega_{0}^{2}\right) / L
$$

where $\omega_{m}=\left(L_{m} C_{m}\right)^{-1 / 2}$. In addition, it also has

$$
M_{S 2}(\omega)=k\left(\omega_{0}\right) \omega_{0}\left(\omega^{2}-\omega_{m}^{2}\right) /\left[\omega\left(\omega_{0}^{2}-\omega_{m}^{2}\right)\right]
$$

Here, it can be noticed that MC coefficient is relative to frequency. It can be either positive or negative which can be considered separately for predigestion. Taking (5) into (2), the position of the TZ related to frequency is written as

$$
\Omega=\frac{\omega / \omega_{0}-\omega_{0} / \omega}{F B W}=\frac{M_{S 1} M_{12}\left[\omega\left(\omega_{0}^{2}-\omega_{m}^{2}\right)\right]}{k\left(\omega_{0}\right) \omega_{0}\left(\omega^{2}-\omega_{m}^{2}\right)}-M_{11}
$$

Here, it can be seen from (2) that conventional modified trisection (MT) without MC can obtain only one transmission zero below or above its passband. By introducing MC with frequency-variant coupling to MT, namely, constructing MCMT, another controllable TZ will be generated in 
the stopband.

To validate above mentioned topology, as shown in Fig. 2, two multilayer SIW filters with MCMT are proposed, which are called the Filters I and II for ease of expatiation. They are both have three metal layers on LTCC substrate, namely, one is on the top, another is on the middle and the last one with all metal is on the bottom, respectively. The difference between two filters is that the positions of the first TZ generated by MT are located above (Filters I) and below (Filters II) the passband, respectively. Here, changing the coupling manner of $M_{S 1}$ from positive (magnetic) coupling to negative (electric) one could move the first TZ from upper to lower stopband conveniently. The positive coupling between the source and resonator 1 in Filter $\mathrm{I}$ is realized by placing the inductive rectangular slots near the sidewalls, where the magnetic field is the strongest. Similarly, to gain a negative coupling in Filter II, the capacitive circular slot is placed in the position between resonator 1 and source, where the electric field is the strongest. Moreover, an interdigital slot-line (ISL) on the top metal layer is implemented to introduce MC between source and resonator 2. Under this circumstance, an additional TZ in both Filters I and II can be obtained with introducing MC. Meanwhile, the values of electric and magnetic coupling of MC are mainly controlled by the length $\left(I_{1}\right)$ of

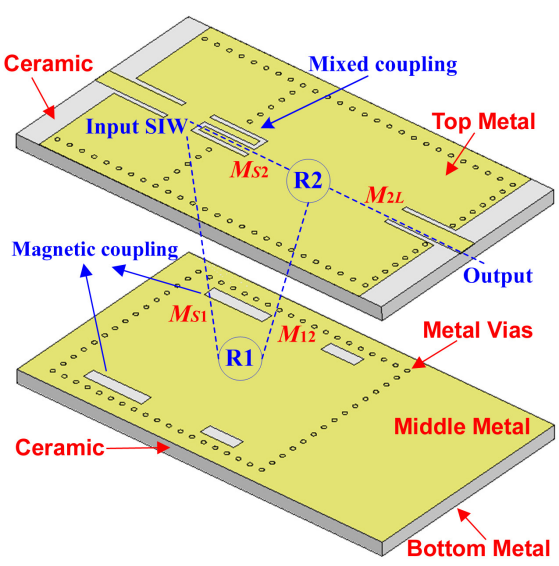

(a)

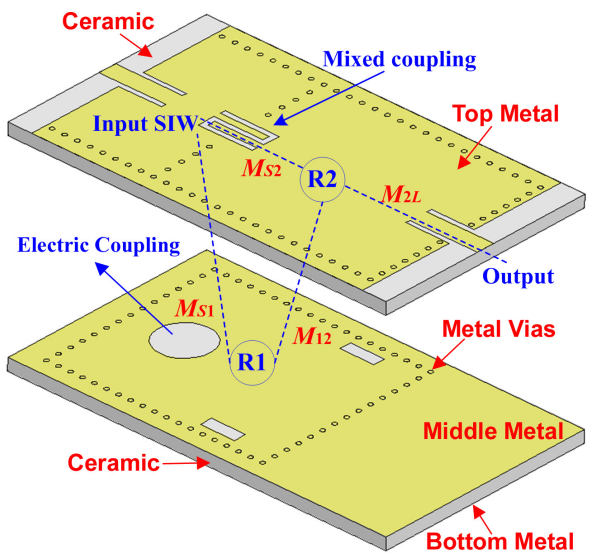

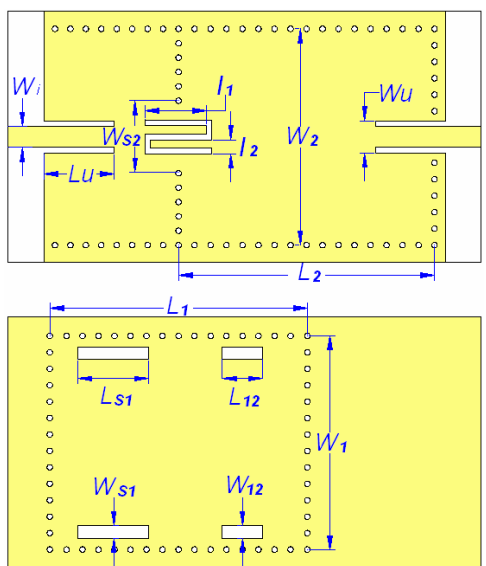

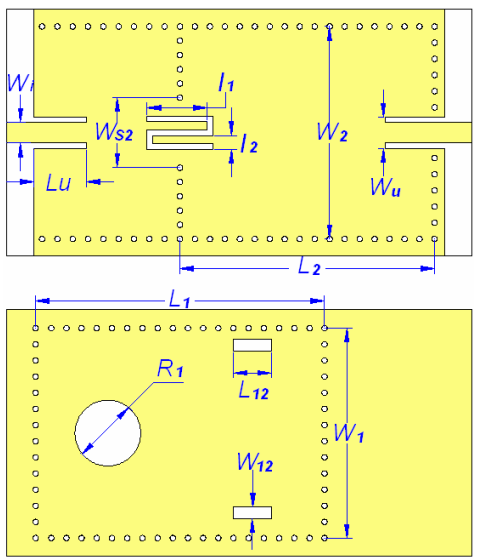

(b)

Fig. 2. 3D overviews and geometric configurations of proposed SIW filters with MCMT: (a) Filter I and (b) Filter II. 

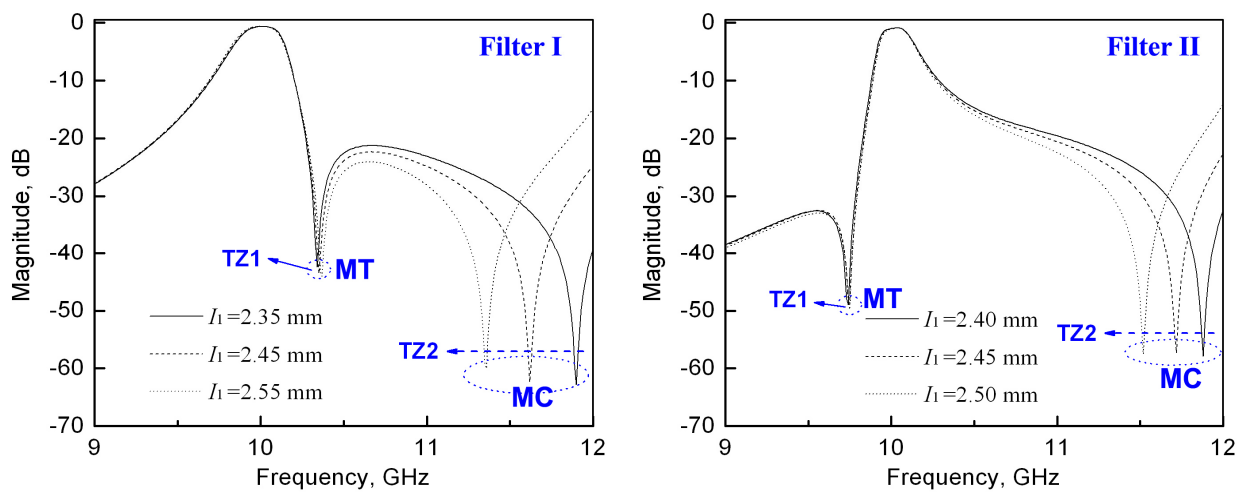

(a)
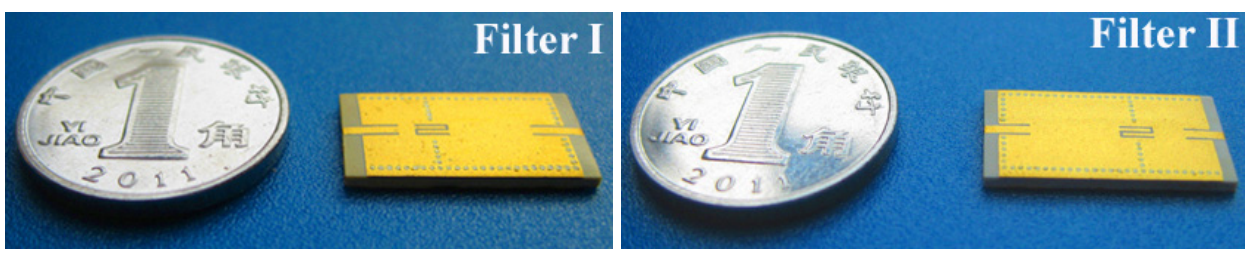

(b)
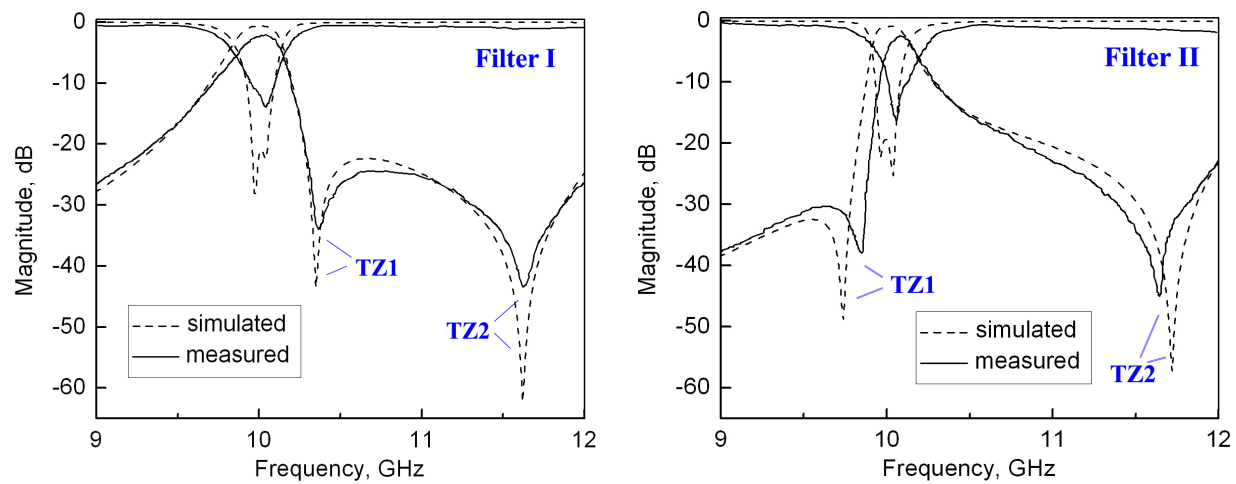

(c)

Fig. 3. Proposed Filters I and II with ten-layer substrate using Ferro-A6M with dielectric constant of $\varepsilon_{r}=$ 5.9, loss tangent of 0.0015 and a single layer thickness of $0.096 \mathrm{~mm}$ : (a) simulated response with dimensions in Table I except different values of $I_{1}$, (b) photographs of fabricated samples, and (c) comparisons of simulated and measured results.

ISL or width of $\mathrm{W}_{S 2}$. Fig. 3 (a) shows the simulated frequency response with dimensions in Table I except different values of $I_{1}$. As an additional TZ caused by MC, the TZ2 is much more sensitive to the values of $I_{1}$ which is distinguished from the TZ1. With increasing the values of $I_{1}$, namely, adding coupling capacitance $C_{m}$, the electric coupling part $\left(C / C_{m}\right)$ of the mixed coupling will become weaker. As shown in Fig. 3(a), the increase of $C_{m}$ will result in the decrease of $\omega_{m}$, and the TZ2 have a tendency to move toward lower frequencies.

\section{Fabrication and measurement}

To demonstrate the application of above-mentioned structures, the proposed filters are fabricated on an ten-layer substrate using LTCC material Ferro-A 6 with relative dielectric constant of $\varepsilon_{r}=5.9$, loss tangent 
Table I. Geometric parameters of Filters I and II (unit: $\mathrm{mm})$.

\begin{tabular}{ccccccccc}
\hline Symbol & $L_{1}$ & $L_{2}$ & $L_{S 1}$ & $L_{12}$ & $L_{u}$ & $I_{1}$ & $I_{2}$ & $R_{1}$ \\
\hline Filter I & 9.26 & 9.22 & 2.55 & 1.45 & 2.5 & 2.45 & 0.5 & -- \\
\hline Filter II & 10.56 & 9.32 & -- & 1.42 & 1.9 & 2.45 & 0.5 & 2.4 \\
\hline Symbol & $W_{1}$ & $W_{2}$ & $W_{S 1}$ & $W_{12}$ & $W_{u}$ & $W_{S 2}$ & $W_{i}$ & -- \\
\hline Filter I & 7.68 & 7.78 & 0.45 & 0.45 & 1.15 & 2.62 & 0.75 & -- \\
\hline Filter II & 7.65 & 7.78 & -- & 0.45 & 1.15 & 2.55 & 0.75 & -- \\
\hline
\end{tabular}

of 0.0015 and a single layer thickness of $0.096 \mathrm{~mm}$. Every SIW unit is built on a five-layer substrate. Table I gives the final geometric parameters of the proposed filters.

By virtue of the $3 \mathrm{D}$ structure and flexible coupling manner, the overall sizes of both fabricated filters are only $17 \times 9 \times 0.97 \mathrm{~mm}^{3}$, which are illustrated in Fig. 3(b). The measured and simulated results are plotted in Fig. 3(c). The measured response of Filter I is in good agreement with the simulation, and it exhibits a central frequency of $10.03 \mathrm{GHz}$ with a $3 \mathrm{~dB}$ fractional bandwidth of $2.9 \%$, minimum passband insertion loss of $2.36 \mathrm{~dB}$, in-band return loss greater than $12 \mathrm{~dB}$. Two TZs are located at $10.3 \mathrm{GHz}$ with $34.6 \mathrm{~dB}$ attenuation and $11.6 \mathrm{GHz}$ with $43.8 \mathrm{~dB}$ attenuation, respectively. Moreover, the measured fractional bandwidth of Filter II is $1.87 \%$ at the central frequency of $10.06 \mathrm{GHz}$. The minimum passband insertion loss is $2.45 \mathrm{~dB}$ and the in-band return loss is greater than $12 \mathrm{~dB}$. Two TZs are located at $9.85 \mathrm{GHz}$ with $39.1 \mathrm{~dB}$ attenuation and $11.6 \mathrm{GHz}$ with $44.3 \mathrm{~dB}$ attenuation. As can be seen, the measured result of Filter II agrees with the simulated one except a little discrepancy in the in-band insertion loss and a small frequency shift. These are mainly caused by manufacturing tolerance of LTCC process during the fabricated process of Filter II, including conductor and dielectric loss, wrinkles of printing conductor surface and edge, and co-fireability of conductor and ceramic material. Particularly, slight offset in the via-filing process makes the actual sizes of resonator cavities little smaller than the designed ones, which is yet another possible factor influencing the frequency shift.

\section{Conclusion}

Novel MCMT and its applications to SIW filters are presented. By implementing MC into MT, the proposed MCMT not only reduce one resonator but also adds a TZ to improve selectivity. Two SIW filters are designed and fabricated using LTCC technology to validate the effectiveness of the proposed structure, with good agreement obtained between their simulated and measured results. Furthermore, in virtue of flexible coupling manner of multilayer structure, the filters exhibit the merits of both miniature size and good selectivity.

\section{Acknowledgements}

This work was supported by National Natural Science Foundation of China (Grant Nos.: 511720346, 1201001), and Fundamental Research Funds for the Central Universities of China (Grant No.: ZYGX2011J132). 